\title{
736.
}

\section{APPLICATION OF THE NEWTON-FOURIER METHOD TO AN IMAGINARY ROOT OF AN EQUATION.}

[From the Quarterly Journal of Pure and Applied Mathematics, vol. xvI. (1879), pp. $179-185$.

I CONSIDER only the most simple case, that of a quadric equation $x^{2}=n^{2}$, where $n^{2}$ is a given imaginary quantity, having the square roots $n$, and $-n$; starting from an assumed approximate (imaginary) value $x=a$, we have $(a+h)^{2}=n^{2}$, that is,

$$
a^{2}+2 a h=n^{2}, \quad h=-\frac{a^{2}-n^{2}}{2 a}, \text { and } a+h=\frac{a^{2}+n^{2}}{2 a} ;
$$

that is, the successive values are

$$
a_{1}=\frac{a^{2}+n^{2}}{2 a}, \quad a_{2}=\frac{a_{1}^{2}+n^{2}}{2 a_{1}}, \ldots,
$$

and the question is, under what conditions do we thus approximate to one determinate root (selected out of the two roots at pleasure), say $n$, of the given equation.

The nearness of two values is measured by the modulus of their difference; thus $a$ nearer to $n$, than $a_{1}$ is to $n$, means mod. $(a-n)<\bmod .\left(a_{1}-n\right)$, and so in other cases; in the course of the approximation $a, a_{1}, a_{2}, \ldots$ to $n$, any step, for instance $a$ to $a_{1}$, is regular if $a_{1}$ is nearer to $n$ than $a$ is, but otherwise it is irregular; the approximation is regular if all the steps are regular, and if (after one or more irregular steps) all the subsequent steps are regular, then the approximation becomes regular at the step which is the first of the unbroken series of regular steps.

We do by an approximation, which is ultimately regular, obtain the value $n$, if only the assumed value $a$ is nearer to $n$ than it is to $-n$; or, say, if the condition mod. $(a-n)<\bmod .(a+n)$ is satisfied, and the approximation is regular from the beginning 
if mod. $(a-n)<\frac{2}{3} \bmod . n$, viz. this condition is a sufficient one*; the first step $a$ to $a_{1}$ will moreover be regular under a less stringent condition imposed upon $a$; and it would seem that, without the condition $\bmod .(a-n)<\frac{2}{3} \bmod . n$ being satisfied, the subsequent steps will in some cases be also regular; that is, that the last-mentioned condition is not a necessary condition in order to the approximation being regular from the beginning; it is, however, the necessary and sufficient condition, to be satisfied by the modulus of $a-n$, in order that the approximation may be regular from the beginning. All this will clearly appear from the geometry.

Fig. 1.

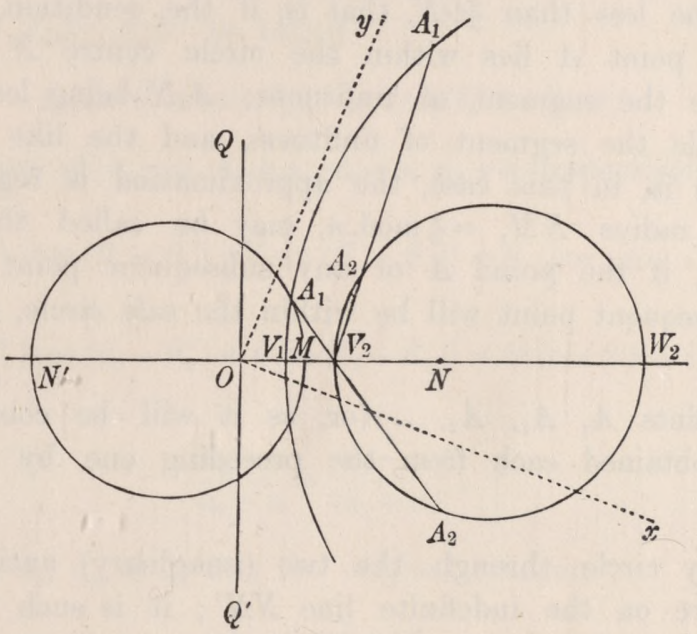

We take $N, N^{\prime}$ (fig. 1 ) to represent the values $n,-n$; and similarly $A, A_{1}$, \&. to represent the quantities $a, a_{1}, \ldots$; we have then

$$
A N=\bmod .(a-n), \quad A_{1} N=\bmod .\left(a_{1}-n\right) \ldots,
$$

so that the approximation is measured by the approach of the points $A, A_{1}$ to $N$. The line $N N^{\prime}$ joining the points $N, N^{\prime}$ passes through, and is bisected at, the origin $O$; drawing then $Q Q^{\prime}$ through $O$ at right angles to $N N^{\prime}$ the condition

$$
\text { mod. }(a-n)<\bmod .(a+n)
$$

means that the point $A$, which represents the imaginary quantity $a$, lies on the $N$-side of $Q Q^{\prime}$, and it will be assumed throughout that this is so. Take now on the line $O N, O M=\frac{1}{3} O N$, and on $N^{\prime} M$ as diameter, describe a circle, which may be called the "circle of unfitness"; regarding as an area the segment hereof which lies on the $N$-side of $Q Q^{\prime}$, say this is the "segment of unfitness." It will be shown that if according as $A$ is situate inside, on the boundary of, or outside the segment of

* In the Smith's Prize Examination, Jan. 28, 1879, I gave the theorem under the following form : "If $a, n$ are imaginary quantities, the latter of them given, and the former assumed at pleasure, subject only to the condition mod. $(a-n)<\frac{2}{3}$ mod. $n$; then if $a_{1}=\frac{a^{2}+n^{2}}{2 a}, a_{2}=\frac{a_{1}^{2}+n^{2}}{2 a_{1}}$, \&c., show that the terms $a, a_{1}, a_{2}, \ldots$ will converge to the limit $n . "$ This is strictly true, but it would have been better to say "will converge regularly." 
unfitness, $A_{1} N$ will be greater than, equal to, or less than $A N$. It may be added that, if $A$ be within or upon the boundary of the segment of unfitness, then $A_{1}$ will be outside it, but this by no means hinders that the next point $A_{2}$, or some later point, shall be within the segment of unfitness; and, further, that when $A$ is outside the segment of unfitness, then the next point $A_{2}$, or some later point, may very well be within the segment of unfitness; the conclusion is, that $A$ being inside the segment of unfitness, $A_{1} N$ is less than $A N$, but that it does not thence follow that $A_{2} N$ is less than $A_{1} N, A_{3} N$ than $A_{2} N, \ldots$; the approximation although regular at the first step, may then, or afterwards, for a step or steps, cease to be regular.

If, however, $A N$ be less than $\frac{2}{3} O N$, that is, if the condition mod. $(a-n)<\frac{2}{3} \bmod . n$ be satisfied, then the point $A$ lies within the circle centre $N$ and radius $N M$, and is consequently outside the segment of unfitness; $A_{1} N$ being less than $A N$, the point $A_{1}$ is a fortiori outside the segment of unfitness, and the like for all the subsequent points $A_{2}, A_{3}, \ldots$, that is, in this case, the approximation is regular throughout. The circle, centre $N$, and radius $N M,=\frac{2}{3} \bmod . n$, may be called the "safe circle"; and the conclusion is that, if the point $A$ or any subsequent point be within the safe circle, then every subsequent point will be within the safe circle, and the approximation will be regular.

The successive points $A, A_{1}, A_{2}, \ldots$ (or, as it will be convenient to call them, $\left.A_{1}, A_{2}, \ldots\right)$ may be obtained each from the preceding one by a simple geometrical construction.

I recall that any circle through the two (imaginary) antipoints of $N, N^{\prime}$ is a circle having its centre on the indefinite line $N N^{\prime}$; it is such that the ratio of the distances of a point thereof from the points $N, N^{\prime}$ respectively has a certain constant value, viz. for the circles with which we are iere alone concerned, those which lie on the $N$-side of $Q Q^{\prime}$, the centres lie beyond the point $N$ (further away, that is, from 0 ), and the values of the ratio, distance from $N$ to distance from $N^{\prime}$, are less unity.

Starting then from the given point $A_{1}$, for which this ratio $A_{1} N: A_{1} N^{\prime}$ has a given value, suppose $A_{1} N=k A_{1} N^{\prime}$, we describe a first circle (passing of course through $A_{1}$ ) for each point of which this ratio has the value $k$; - let the diameter of this circle be $V_{1} W_{1}, V_{1}$ being the extremity between $O$ and $N, W_{1}$ (not shown in the figure), that beyond $N$; we then describe a second circle, for which the ratio is $=k^{2}$; let its diameter be $V_{2} W_{2}, V_{2}$ being the extremity between $O$ and $N$ (or say between $V_{1}$ and $N$ ), $W_{2}$, that beyond $N$ (or say between $N$ and $W_{1}$ ); the point $A_{2}$ lies on this second circle, and is determined as the single intersection of the line $V_{2} A_{1}$ with the second circle. And of course drawing a third circle, for which the ratio is $=k^{4}$, on the diameter $V_{3} W_{3}$, then $A_{3}$ lies on the third circle, and is the intersection with it of the line $V_{3} A_{2}$, and so on; the radii of the successive circles diminish very rapidly, their centres, in like manner, continually approaching the point $N$; hence, the points $A_{1}, A_{2}, A_{3}, \ldots$, which lie on the several circles respectively approximate, and that very rapidly, to the point $O$. But by what precedes, if, for instance, the point $A_{1}$ be within the segment of unfitness, then also some of the subsequent points may be within the segment of unfitness, and for each point $A_{p}$, 
for which this is the case, the next point $A_{p+1}$ is at a greater distance, so that $N A_{p+1}>N A_{p}$; it is, however, clear that we always arrive at a point $A_{q}$, such that $N A_{q}<\frac{2}{3} O N$, and so soon as such a point is arrived at the approximation becomes regular.

The point $A_{2}$ determined from $A_{1}$, as above, is a point such that the subtended angle $N A_{2} N^{\prime}$ is = twice the subtended angle $N A_{1} N^{\prime}$; or calling the latter angle $\phi$, the former is $=2 \phi$. It is, in fact, this property which gives rise to the construction; for let the values of $A_{1} N, A_{1} N^{\prime}$, regarded as imaginary quantities, be called for a moment

$$
\rho_{1}\left(\cos \theta_{1}+i \sin \theta_{1}\right), \quad \rho_{1}^{\prime}\left(\cos \theta_{1}^{\prime}+i \sin \theta_{1}^{\prime}\right) ;
$$

and, similarly, those of $A_{2} N, A_{2} N^{\prime}$ be called

$$
\rho_{2}\left(\cos \theta_{2}+i \sin \theta_{2}\right), \quad \rho_{2}^{\prime}\left(\cos \theta_{2}^{\prime}+i \sin \theta_{2}^{\prime}\right) ;
$$

then these are the values of $a_{1}-n, a_{1}+n, a_{2}-n, a_{2}+n$ respectively, or we have

$$
\begin{aligned}
& \frac{a_{1}-n}{a_{1}+n}=\frac{\rho_{1}}{\rho_{1}^{\prime}}\left\{\cos \left(\theta_{1}-\theta_{1}^{\prime}\right)+i \sin \left(\theta_{1}-\theta_{1}^{\prime}\right)\right\}=k(\cos \phi+i \sin \phi), \\
& \frac{a_{2}-n}{a_{2}+n}=\frac{\rho_{2}}{\rho_{2}^{\prime}}\left\{\cos \left(\theta_{2}-\theta_{2}^{\prime}\right)+i \sin \left(\theta_{2}-\theta_{2}^{\prime}\right)\right\}=k^{2}(\cos 2 \phi+i \sin 2 \phi),
\end{aligned}
$$

that is,

$$
\frac{a_{2}-n}{a_{2}+n}=\left(\frac{a_{1}-n}{a_{1}+n}\right)^{2}
$$

which relation between $a_{2}, a_{1}$ is in fact the original relation

$$
a_{2}=\frac{a_{1}^{2}+n^{2}}{2 a_{1}}
$$

and, conversely, $a_{1}, a_{2}$ being thus connected, then the representative $A_{2}$ is obtained from the representative point $A_{1}$ by the foregoing geometrical construction.

I give the analytical proofs; we may without loss of generality take, and it is convenient to do so, the axis of $x$ as coinciding with the line $O N$, and to put also $O N=1$. We then in place of the original coordinates $x, y$ of any point take the new coordinates $k, \phi$ which are'such that

$$
\begin{aligned}
& \frac{x+i y-1}{x+i y+1}=k e^{i \phi}, \\
& \frac{x-i y-1}{x-i y+1}=k e^{-i \phi}
\end{aligned}
$$

equations which may also be written

$$
\begin{aligned}
& (x-1)^{2}+y^{2}=k^{2}\left[(x+1)^{2}+y^{2}\right], \\
& x^{2}+(y-i)^{2}=e^{-2 i \phi}\left[x^{2}+(y+i)^{2}\right] ;
\end{aligned}
$$

or, what is the same thing,

$$
x^{2}+y^{2}-1-2 y \cot \phi=0,
$$


where of course the equation with $k$ shows that $k$ is equal to the ratio of the distances of the point from the points $N, N^{\prime}$ respectively, and the equation in $\phi$, taken in the second form, shows that $\phi$ is the angle subtended at the point by $N, N^{\prime}$.

It is sometimes convenient to write $k e^{i \phi}, k e^{-2 i \phi}=p, q$ respectively; we then have

$$
p q=k^{2}, \quad x+i y=\frac{1+p}{1-p}, \quad x-i y=\frac{1+q}{1-q} .
$$

Suppose for a moment that we have $\left(p_{1}, q_{1}\right),\left(p_{2}, q_{2}\right),\left(p_{3}, q_{3}\right)$ as the $(p, q)$ coordinates of any three points, the condition that these three points may lie in a line, is given in the form, determinant $=0$, where each line of the determinant is of the form

$$
\frac{1+p}{1-p}, \quad \frac{1+q}{1-q}, \quad 1
$$

or, what is the same thing, it is

or, again

viz. the condition is

$$
1-p q+p-q, \quad 1-p q-p+q, \quad 1+p q-p-q,
$$

$$
p q-1, \quad p-q, \quad p+q-2,
$$

$$
\left|\begin{array}{lll}
p_{1} q_{1}-1, & p_{1}-q_{1}, & p_{1}+q_{1}-2 \\
p_{2} q_{2}-1, & p_{2}-q_{2}, & p_{2}+q_{2}-2 \\
p_{3} q_{3}-1, & p_{3}-q_{3}, & p_{3}+q_{3}-2
\end{array}\right|=0
$$

Suppose the $(k, \phi)$ coordinates of the three points are $(l, \alpha),(m, \beta),(n, \gamma)$ respectively; then this equation is

viz. it is

$$
\left|\begin{array}{lll}
l^{2}-1, & l \sin \alpha, & l \cos \alpha-1 \\
m^{2}-1, & m \sin \beta, & m \cos \beta-1 \\
n^{2}-1, & n \sin \gamma, & n \cos \gamma-1
\end{array}\right|=0
$$

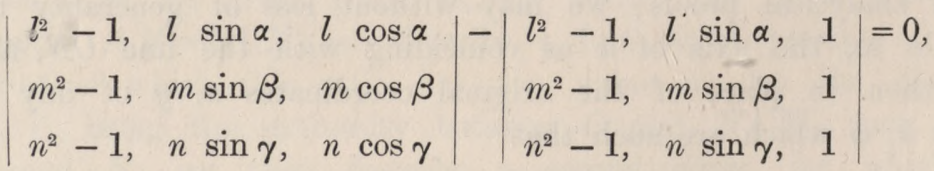

or, what is the same thing, it is

$$
\begin{aligned}
& {\left[\left(l^{2}-1\right) m n \sin (\beta-\gamma)+\left(m^{2}-1\right) n l \sin (\gamma-\alpha)+\left(n^{2}-1\right) l m \sin (\alpha-\beta)\right]} \\
& \quad+\left[\left(m^{2}-n^{2}\right) l \sin \alpha+\left(n^{2}-l^{2}\right) m \sin \beta+\left(l^{2}-m^{2}\right) n \sin \gamma\right]=0 .
\end{aligned}
$$

If in this equation $\gamma$ is put $=\pi$, and $\beta=2 \alpha$, so that $\sin (\alpha-\beta)=-\sin \alpha$, the equation will contain only terms in $\sin \alpha$, and $\sin 2 \alpha$, viz. it will be

that is,

$$
\begin{aligned}
& {\left[\left(m^{2}-n^{2}\right) l+\left(m^{2}-1\right) n l-\left(n^{2}-1\right) l m\right] \sin \alpha} \\
& +\left[-\left(l^{2}-1\right) m n+m\left(n^{2}-l^{2}\right) \quad\right] \sin 2 \alpha=0 \text {, }
\end{aligned}
$$

$$
l(m-1)(n+1)(m-n) \sin \alpha+m(m+1)\left(n-l^{2}\right) \sin 2 \alpha=0,
$$


or, what is the same thing,

$$
(m+1) \sin \alpha\left\{l(n+1)(m-n)+2 m\left(n-l^{2}\right) \cos \alpha\right\}=0,
$$

which is satisfied for any values whatever of $l, m, n$, by a proper value of $\cos \alpha$; and is also satisfied irrespectively of the value of $\alpha$ if only $m=n=l^{2}$; or, writing $k$ instead of $l$, say if $l=k, m=n=k^{2}$; that is, writing also $\phi$ in place of $\alpha$, the three points

$$
(k, \phi),\left(k^{2}, 2 \phi\right) \text { and }\left(k^{2}, \pi\right)
$$

are in a right line; viz. the point $A_{1}$, circle $k$, subtended angle $\phi$; the point $A_{2}$, circle $k^{2}$, subtended angle $2 \phi$; and the point $V_{2}$, same circle, subtended angle $\pi$; are in a right line.

The equation of the circle of unfitness can be obtained more easily in a different manner; but I have thought it worth while to give the investigation by means of the foregoing $(p, q)$ coordinates.

Suppose that $p_{1}, q_{1}$ refer to the point $A_{1}$ : then we have

$$
\left(A_{1} N\right)^{2}=\left(x_{1}-1\right)^{2}+y_{1}^{2}=\left(x_{1}+i y_{1}-1\right)\left(x_{1}-i y_{1}-1\right),=\left(\frac{1+p_{1}}{1-p_{1}}-1\right)\left(\frac{1+q_{1}}{1-q_{1}}-1\right),
$$

that is,

$$
\left(A_{1} N\right)^{2}=\frac{4 p_{1} q_{1}}{1-p_{1} \cdot 1-q_{1}} .
$$

Similarly, if $p_{2}, q_{2}$ refer to the point $A_{2}$, then

$$
\left(A_{2} N\right)^{2}=\frac{4 p_{2} q_{2}}{1-p_{2} \cdot 1-q_{2}}, \quad=\frac{4 p_{1}^{2} q_{1}^{2}}{1-p_{1}^{2} \cdot 1-q_{1}^{2}},
$$

since $p_{2}, q_{2}=p_{1}{ }^{2}, q_{1}{ }^{2}$. The two are equal if

that is,

$$
\left(1+p_{1}\right)\left(1+q_{1}\right)=p_{1} q_{1}
$$

$$
p_{1}+q_{1}+1=0 .
$$

Writing for a moment $x_{1}+i y_{1}=\xi, x_{1}-i y_{1}=\eta$, we have

and the equation is

$$
p_{1}, q_{1}=\frac{\xi-1}{\xi+1}, \frac{\eta-1}{\eta+1},
$$

that is,

$$
\frac{\xi-1}{\xi+1}+\frac{\eta-1}{\eta+1}+1=0
$$

$$
3 \xi \eta+\xi+\eta-1=0
$$

or substituting for $\xi, \eta$ their values, the equation is

that is,

$$
3\left(x_{1}^{2}+y_{1}^{2}\right)+2 x_{1}-1=0,
$$

$$
\left(x_{1}+1\right)\left(x_{1}-\frac{1}{3}\right)+y_{1}^{2}=0 \text {, }
$$


the equation of a circle on the diameter $N^{\prime} M$, which is, in fact, the before-mentioned circle of unfitness; viz. $A_{1}$ being on the circumference of this circle, or say on the boundary of the segment of unfitness, then $A_{1} N=A_{2} N$; whence also, according as $A_{1}$ is inside or outside the segment, $A_{1} N<A_{2} N$ or $>A_{2} N$.

Suppose $A_{1}$ to be on the circle, that is, $p_{1}+q_{1}+1=0$; it is easy to show that the locus of $A_{2}$ is also a circle. We have in fact $\left(p_{1}+q_{1}\right)^{2}-1=0$, that is,

$$
p_{2}+q_{2}+2 k^{2}-1=0
$$

or say

$$
\frac{\xi-1}{\xi+1}+\frac{\eta-1}{\eta+1}+2 k^{2}-1=0
$$

viz. this is

$$
\left(2 k^{2}+1\right) \xi \eta+\left(2 k^{2}-1\right)(\xi+\eta)+2 k^{2}-3=0,
$$

that is,

$$
x_{2}^{2}+y_{2}^{2}+\frac{2 k^{2}-1}{2 k^{2}+1} 2 x_{2}+\frac{2 k^{2}-3}{2 k^{2}+1}=0
$$

or finally

$$
\left(x_{2}+1\right)\left(x_{2}-\frac{3-2 k^{2}}{1+2 k^{2}}\right)+y_{2}^{2}=0 \text {. }
$$

Measuring off from $O$ in the direction of $O N$, a distance $O S=\frac{3-2 k^{2}}{1+2 k^{2}}$ (always $>\frac{1}{3}$, since $k^{2}<1$ ), the circle in question is that on the diameter $N^{\prime} S$; this is a circle touching at $N^{\prime}$, and containing within it the circle of unfitness; if $k=1$ (that is, for $A_{1}$ on the line $Q Q^{\prime}$ ) it becomes identical with the circle of unfitness, but except in this limiting case it does not meet the circle of unfitness in any point on the $N$-side of $Q Q^{\prime}$, that is, $A_{1}$ being on the boundary of the segment of unfitness $A_{2}$ is never on this boundary; and it thus appears that $A_{1}$ being inside the segment, $A_{2}$ is always outside the segment.

It is to be further noticed, that we have

$$
\frac{\left(A_{1} N\right)^{2}}{\left(A_{2} N\right)^{2}}=\frac{1+p_{1} \cdot 1+q_{1}}{p_{1} q_{1}}
$$

or

$$
\frac{\left(A_{1} N\right)^{2}}{\left(A_{2} N\right)^{2}}-1=\frac{1+p_{1}+q_{1}}{k^{2}}=\frac{3 \xi \eta+\xi+\eta-1}{k^{2}(\xi \eta+\xi+\eta+1)},=\frac{3\left(x_{1}^{2}+y_{1}^{2}+\frac{2}{3} x_{1}-\frac{1}{3}\right)}{k^{2}\left[\left(x_{1}+1\right)^{2}+y_{1}^{2}\right]},
$$

that is,

$$
\frac{\left(A_{1} N\right)^{2}}{\left(A_{2} N\right)^{2}}-1=\frac{3 T^{2}}{k^{2}\left(A_{1} N\right)^{2}},
$$

where $T$ is the tangential distance of $A_{1}$ from the circle of unfitness; there should, it appears to me, be some more elegant formula for the ratio $A_{1} N \div A_{2} N$ which determines whether the step is regular or irregular. 
It is worth noticing how the conditions

$$
\text { mod. }(a-n)<\bmod .(a+n) \text { and mod. }(a-n)<\frac{2}{3} \bmod . n \text {, }
$$

present themselves in the real theory. Making the usual construction by means of the parabola $y=x^{2}$, the first condition means that the point $A$ must be taken on the $N$-side of $O$ (fig. 2); the second that, in order to the regularity of the approxi-

Fig. 2.

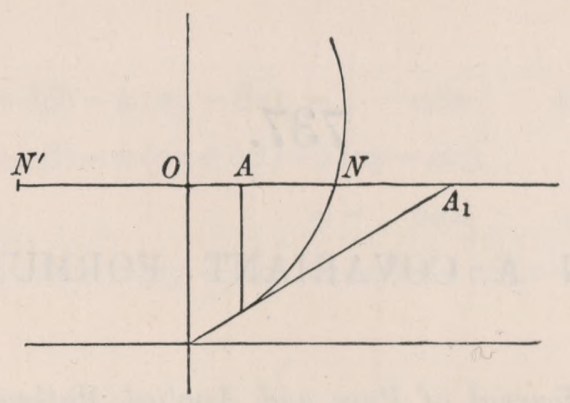

mation, $A$ must be taken at a distance from $O>\frac{1}{3} O N$; in fact, if (as in the figure) $O A=\frac{1}{3} O N$, then $A N=N A_{1}$, or the point $A_{1}$ is at an equal distance with $A$ from $N$; and thence, according as $O A$ is greater or less than $\frac{1}{3} O N$, the point $A_{1}$ is nearer or further than $A$ to or from $N$. 